\title{
PAPER \\ Practical Design Methodology of Mode-Conversion-Free Tightly Coupled Asymmetrically Tapered Bend for High-Density Differential Wiring*
}

\author{
Chenyu WANG $^{\dagger \mathrm{a})}$, Member, Kengo IOKIBE ${ }^{\dagger \mathrm{b})}$, and Yoshitaka TOYOTA ${ }^{\dagger \mathrm{c})}$, Senior Members
}

\begin{abstract}
SUMMARY The plain bend in a pair of differential transmission lines causes a path difference, which leads to differential-to-common mode conversion due to the phase difference. This conversion can cause serious common-mode noise issues. We previously proposed a tightly coupled asymmetrically tapered bend to suppress forward differential-to-common mode conversion and derived the constraint conditions for high-density wiring. To provide sufficient suppression of mode conversion, however, the additional correction was required to make the effective path difference vanish. This paper proposes a practical and straightforward design methodology by using a very tightly coupled bend (decreasing the line width and the line separation of the tightly coupled bend). Full-wave simulations below $20 \mathrm{GHz}$ demonstrated that sufficient suppression of the forward differential-to-common mode conversion is successfully achieved as designed. Measurements showed that our design methodology is effective. key words: differential transmission lines, mode-conversion, high-density wiring, asymmetric tapers, design methodology
\end{abstract}

\section{Introduction}

Differential signaling has become required in high-speed digital systems due to their high immunity to noise, low crosstalk, and low electromagnetic interference, and it is generally used for high-speed interconnector interfaces, such as PCI Express, USB3.0, HDMI, and so on. For differential signaling on printed circuit boards (PCBs), a pair of symmetric coupled transmission lines is used. However, in practice, discontinuities in the trace layout are inevitable and generate asymmetry. For example, there can be a path difference between the inner and outer lines of differential transmission lines with asymmetrical layouts, such as those with bend discontinuities, as shown in Fig. 1(a). Thus, the output phase difference (between Ports 3 and 4) between Line \#1 and Line \#2 may not maintain a constant value at $180^{\circ}$. The path difference causes a mode conversion from the differential-to-common mode, and this conversion can cause serious common-mode noise issues and degrade signal integrity (SI) [1], [2].

Manuscript received April 9, 2020.

Manuscript revised August 3, 2020.

Manuscript publicized September 15, 2020.

$\dagger$ The authors are with the Graduate School of Natural Science and Technology, Okayama University, Okayama-shi, 7008530 Japan.

*This paper was presented at APEMC 2016/Shenzhen.

a) E-mail: p1kw7v6x@s.okayama-u.ac.jp

b) E-mail: iokibe@okayama-u.ac.jp

c)E-mail: toyota@okayama-u.ac.jp

DOI: 10.1587/transcom.2020EBP3056

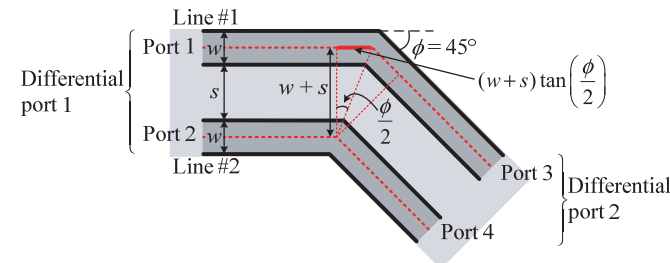

(a) Conventional bend

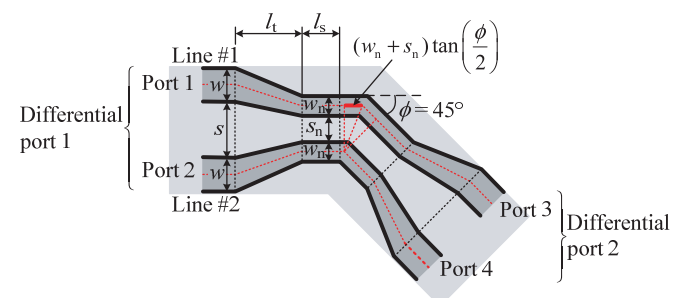

(b) Tightly coupled symmetrically tapered bend [10]-[13]

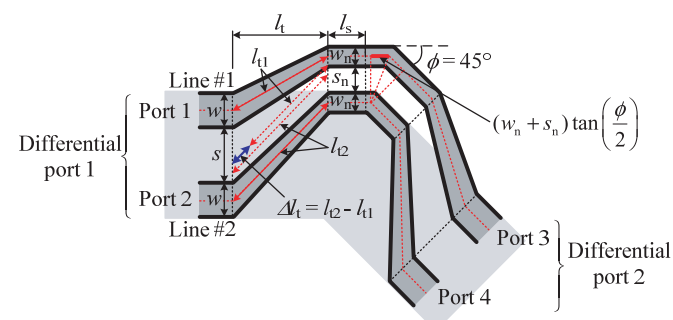

(c) Tightly coupled asymmetrically tapered bend [14], [15]

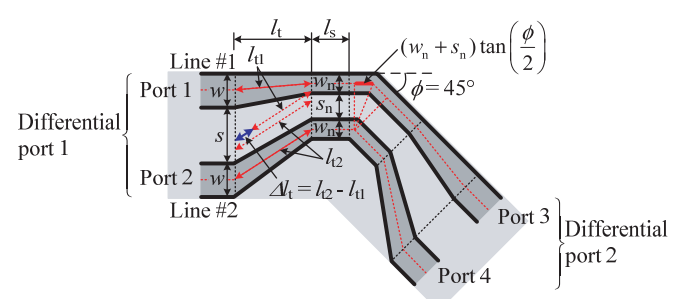

(d) Tightly coupled asymmetrically tapered bend for highdensity wiring [16], [17]

Fig. 1 45-degree-angle bends of differential transmission lines treated in this paper.

In recent years, several researchers have proposed various methods to suppress the differential-to-common mode conversion caused by differential transmission line bend structures. For example, using a square patch for right-angle 
bends and a fan-shaped patch for 45-degree-angle bends to create a compensation capacitance in the inner line [1], using a short-circuited coupled line for compensation inductance [3], using a slow-wave structure scheme [4], [5], placing a guard trace and a stub structure with narrowed inner line [6], [7], using surface mounted device capacitors for compensation capacitance of the asymmetric coupled lines inner lines [8], using mushroom structures with asymmetric coupled lines [9]. These proposed structures can suppress the differential-to-common mode conversion. However, these bend structures have protruding structures that reduce wiring density and exhibit a larger differential-mode reflection than the conventional bend structure. The progress of modern technology has led to an increasing trend toward higher speed, high-density wiring. Therefore, these proposed structures are difficult to apply to the next generation of miniaturized high-density wiring PCBs.

In this study, we focus on suppressing the differential-tocommon mode conversion by decrease the path difference. In keeping the differential-mode characteristic impedance constant, tightly coupled differential transmission lines shorten the path difference by decrease the line width and line separation, while a narrow line width increases propagation loss due to the skin effect. In [10]-[13], a tightly coupled symmetrically tapered bend shown in Fig. 1(b) has been proposed to simultaneously achieve lower propagation loss due to weakly coupled straight lines and lower differential-tocommon mode conversion due to the tightly coupled bend area. However, although the tightly coupled bend shortens the path difference, the difference is not completely removed.

To compensate for the remaining path difference in the tightly coupled bend, we previously proposed a tightly coupled asymmetrically tapered bend to suppress the differential-to-common mode conversion [14], [15], as shown in Fig. 1(c). The concept of the proposed bend is to compensate for the path difference remaining in the bend by introducing asymmetric tapers. As shown in Fig. 1(c), the total taper length of Line \#1 is set to be shorter than that of Line \#2, and the path difference of the tightly coupled bend can be removed by adjusting the length of the asymmetric taper. However, the proposed bend structure was not limited to within the area of the conventional bend, which can also degrade high-density wiring on PCBs.

Therefore, we investigated the essential design methodology of our tightly coupled asymmetrically tapered bend to limit it to within the area of the original classic bend through full-wave simulation and clarified its required constraint conditions, as shown in Fig. 1(d) [16], [17]. In [15]-[17], the geometrical path difference is substituted for the effective path difference. But in practice, an additional correction (the geometrical path difference set to a negative value) is required to make the effective path difference vanish. In [17], we did not find the practical and straightforward design methodology by using the geometrical path difference, and we did not carry out the evaluation through the fabrication and measurement neither. In this paper, we develop practical and straightforward design methodology based on the geometrical path difference by decreasing the line width and line separation of the tightly coupled bend and also validate the design methodology by not only full-wave simulation but also measurement.

In this paper, we first explain the essential design methodology of our tightly coupled asymmetrically tapered bend to limit it to within the area of the conventional bend and its required constraint conditions proposed in [16], [17]. Then, we propose the use of a very tightly coupled bend (the smallest possible values of the line width and line separation of the tightly coupled bend) to make the effective path difference equal to the geometric path difference for sufficient suppression of mode conversion, which leads to the achievement of practical and straightforward design methodology. The design methodology is validated using the results obtained by not only a full-wave simulation but also measurement.

\section{Tightly Coupled Asymmetrically Tapered Bend for High-Density Wiring}

In this section, in accordance with the following structural parameters, we explain the practical and straightforward design methodology of our bend structure shown in Fig. 1(d) to limit it to within the area of the conventional bend as a light gray area.

- $w$ : line width

- $s$ : separation between differential transmission lines

- $w_{\mathrm{n}}$ : line width of tightly coupled bend

- $s_{\mathrm{n}}$ : separation between differential transmission lines of tightly coupled bend

- $l_{\mathrm{t}}:$ taper length

- $l_{\mathrm{t} 1}:$ taper's center-line length of Line \#1

- $l_{\mathrm{t} 2}:$ taper's center-line length of Line \#2

- $l_{\mathrm{s}}$ : length of straight part between taper and tightly coupled bend

- $l_{\mathrm{dg}}$ : geometrical path difference

- $l_{\mathrm{d}}$ : effective path difference

- $\Delta l_{\mathrm{t}}$ : compensation amount

\subsection{Geometrical Path Difference and Effective Path Differ-} ence

The differential-to-common mode conversion is strongly related to the effective path difference, but it is challenging to define the effective path appropriately. In [15], [17], the geometrical path difference $l_{\mathrm{dg}}$ is substituted for the effective path difference $l_{\mathrm{d}}$. In Fig. 1, the geometrical path difference $l_{\mathrm{dg}}$ is defined as the subtraction of the center-line length of Line \#1 from that of Line \#2, which is represented as a short solid red line. The figures also show the geometrical path difference of three types of bends depending on the bend angle $\phi$. First, the geometrical path difference of the conventional bend shown in Fig. 1(a) is given as

$$
l_{\mathrm{dg}}=2(w+s) \tan \left(\frac{\phi}{2}\right) \text {. }
$$


Next, $l_{\mathrm{dg}}$ of the tightly coupled symmetrically tapered bend [10]-[13] shown in Fig. 1(b) is given by replacing $w+$ $s$ with $w_{\mathrm{n}}+s_{\mathrm{n}}$ in (1). The tightly coupled symmetrically tapered bend shortens the geometrical path difference because $w_{\mathrm{n}}<w$ and $s_{\mathrm{n}}<s$, but the geometrical path difference of $2\left(s_{\mathrm{n}}+w_{\mathrm{n}}\right) \tan (\phi / 2)$ still remains.

Finally, our tightly coupled bend with asymmetric tapers shown in Figs. 1(c) and (d) have oblique tapers that can compensate for $l_{\mathrm{dg}}$ that remains in the tightly coupled symmetrically tapered bend. Extending the taper of Line \#2 and shortening that of Line \#1 results in the compensation amount $\Delta l_{\mathrm{t}}=l_{\mathrm{t} 2}-l_{\mathrm{t} 1}$, which is represented as a short solid blue double-arrow, where $l_{\mathrm{t} 1}$ and $l_{\mathrm{t} 2}$ are the taper's centerline lengths of Lines \#1 and \#2, respectively. Therefore, $l_{\mathrm{dg}}$ of our tightly coupled asymmetrically tapered bend shown in Figs. 1(c) and (d) is given as

$$
l_{\mathrm{dg}}=2\left\{\left(w_{\mathrm{n}}+s_{\mathrm{n}}\right) \tan \left(\frac{\phi}{2}\right)-\Delta l_{\mathrm{t}}\right\} \text {. }
$$

Consequently, the total geometrical path difference is removed when the taper of Line \#2 is longer by $2\left(s_{\mathrm{n}}+\right.$ $\left.w_{\mathrm{n}}\right) \tan (\phi / 2)$ than that of Line \#1, that is, $\Delta l_{\mathrm{t}}=2\left(s_{\mathrm{n}}+\right.$ $\left.w_{\mathrm{n}}\right) \tan (\phi / 2)$.

Although we defined the geometrical path difference for three different bend structures, the reader should be aware of the limitations of this geometrical path difference. There is one empirically conjectured effect that is worth noting. As shown in Fig. 1(d), we can easily observe that the degree of change in the taper part of Line \#2 is more intense than that of Line \#1. The signal flow in an asymmetrically tapered structure will flow in such a manner that it will deviate from the expected delay on the basis of the center-line length. In Fig. 2(a), consider the arrow line as a component of the signal. Since the signal cuts both corners in the taper of Line $\# 2$, that component of the signal will arrive at the destination slightly earlier than expected, as described in Sect. 4.5 of [18]. Then, we know that in differential transmission lines with asymmetrically tapered structures, the effective path difference may be slightly different than expected. This effect has been seen in full-wave simulations and measurements [14]-[17]. Since our design methodology is based on the geometrical path length, in practice, an additional correction is required to remove the effective path difference.

As shown in Fig. 2(b), we can imagine that the signal flow in an asymmetrically tapered structure will flow in such a manner that it will be close to the expected path (center-line

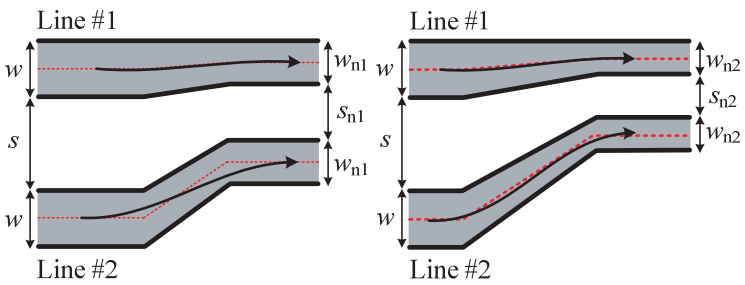

(a) Big difference

(b) Small difference

Fig. 2 Geometrical path and effective path $\left(w_{\mathrm{n} 1}>w_{\mathrm{n} 2}, s_{\mathrm{n} 1}>s_{\mathrm{n} 2}\right)$. length), when $w_{\mathrm{n} 2}<w_{\mathrm{n} 1}$ and $s_{\mathrm{n} 2}<s_{\mathrm{n} 1}$. Therefore, in this paper, we will reduce the difference between the geometric path and the effective path by decreasing $w_{\mathrm{n}}$ and $s_{\mathrm{n}}$ of the tightly coupled bend.

\subsection{Range of Values for $w_{\mathrm{n}}$ and $s_{\mathrm{n}}$}

We now briefly describe our design methodology, which is determined geometrically from the bend structure. First, the range of values for the structural parameters of $w_{\mathrm{n}}$ and $s_{\mathrm{n}}$ are determined when the geometrical path difference $l_{\mathrm{dg}}$ is set to 0 . Once $\phi$ is given, $w_{\mathrm{n}}$ and $s_{\mathrm{n}}$ are determined so that

$$
w_{\mathrm{n}}+s_{\mathrm{n}}<\frac{w+s}{1+\tan (\phi / 2)}
$$

In other words, to form a taper in which the geometrical path difference $l_{\mathrm{dg}}=0$, the line width $w_{\mathrm{n}}$ and line spacing $s_{\mathrm{n}}$ must satisfy the range of (3). In addition, we previously clarified in Sect. 2.1 that our bend structure can reduce the difference between the geometrical path difference $l_{\mathrm{dg}}$ and the effective path difference $l_{\mathrm{d}}$ by adjusting $w_{\mathrm{n}}$ and $s_{\mathrm{n}}$ of the tightly coupled bend. Therefore, when designing the proposed structure, we needed to choose the smallest possible values of $w_{\mathrm{n}}$ and $s_{\mathrm{n}}$. Appendix explains the derivation process of (3).

\subsection{Taper Length $l_{\mathrm{t}}$ for Dense Traces}

Figure 1(d) shows the case in which the outer side of Line $\# 1$ is coincident with that of the conventional bend, and $l_{\mathrm{t}}$ is given as

$$
\begin{aligned}
l_{\mathrm{t}}= & \left\{\left[\frac{\left(w-w_{\mathrm{n}}+s-s_{\mathrm{n}}\right)\left(2 w-2 w_{\mathrm{n}}+s-s_{\mathrm{n}}\right)-\Delta l_{\mathrm{t}}^{2}}{2 \Delta l_{\mathrm{t}}}\right]^{2}\right. \\
& \left.-\frac{\left(w-w_{\mathrm{n}}\right)^{2}}{4}\right\}^{\frac{1}{2}} .
\end{aligned}
$$

Appendix explains the derivation process of (4).

\subsection{Straight Part Length $l_{\mathrm{s}}$ between Taper and Tightly Cou- pled Bend}

The straight part length $l_{\mathrm{s}}$ between the taper and tightly coupled bend significantly increases propagation loss due to the skin effect. However, in Fig. 3, a too-short $l_{\mathrm{s}}$ causes the electromagnetic coupling of the taper area on Line \#2 to increase as shown by the arrows, which changes the characteristic impedance and propagation constants and hence affects the suppression amount of the differential-to-common mode conversion and differential-mode propagation characteristics of the tightly coupled asymmetrically tapered bend. The most important thing in the design of $l_{\mathrm{s}}$ is to select an appropriate value for $l_{\mathrm{p}}$ upon careful consideration of the differential-to-common mode conversion and differentialmode propagation characteristics.

For single-ended lines, the general guidelines expressed 
by the $3 w$ (line separation is 3 times the line width) rule are known [19] because the crosstalk (electromagnetic coupling) can be made sufficiently small. In this paper, for simplicity, $l_{\mathrm{p}} \geq 3 w$ is set as the optimal condition. Its effectiveness has been proven in [16]. There is room for consideration in the calculation of $l_{\mathrm{p}}$ adopted here. This is a topic for future work.

\subsection{Design Methodology}

Figure 4 shows our design methodology of our bend structure for high-density wiring. By using this design, we can easily and quickly design our proposed structure, and this method is suitable for the wiring bend angles commonly used in PCB design.

It should be noted that our design method is based on the geometrical path difference $l_{\mathrm{dg}}$ of 0 . Step 1 involves determining the range of $w_{\mathrm{n}}$ and $s_{\mathrm{n}}$ by calculating (3). As mentioned earlier, small values within the range of values need to be selected for $w_{\mathrm{n}}$ and $s_{\mathrm{n}}$. In the following sections, we focus on the impact of $w_{\mathrm{n}}$ and $s_{\mathrm{n}}$ on the differential-tocommon mode conversion. Step 2 involves determining $l_{\mathrm{t}}$ by calculating (4) to limit the asymmetrically tapered bend within the area of the conventional bend. In Step 3, $l_{\mathrm{s}}$ is determined as referred in Sect. 2.4.

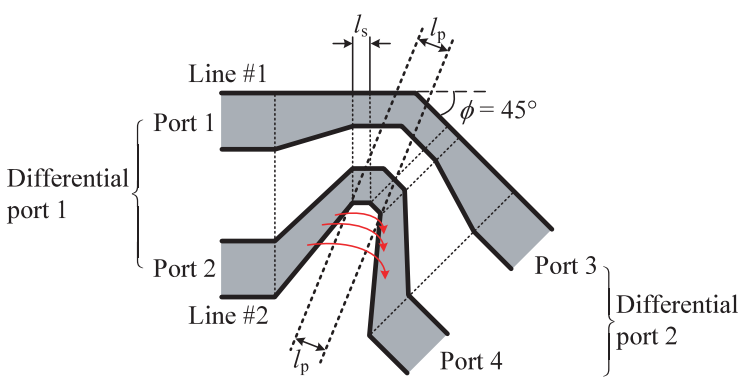

Fig. 3 New issue caused by too small $l_{\mathrm{s}}$.

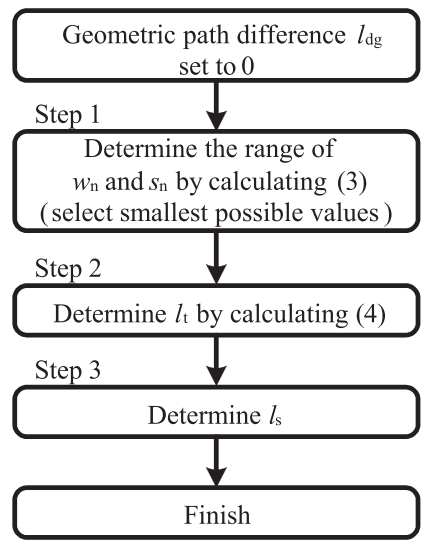

Fig. 4 Design flows of our tightly coupled asymmetrically tapered bend for high-density wiring.

\section{Evaluation of Mode Conversion in Our Bend Struc- tures Based on Proposed Methodology}

In this section, first, we use our design methodology to determine the structural parameters of $w_{\mathrm{n}}, s_{\mathrm{n}}, l_{\mathrm{t}}$, and $l_{\mathrm{s}}$. These determined parameters are used in Sect. 4 for the fabrication test board and measurement. Then, we validate the bend structure formed on the basis of our design methodology by full-wave simulation using a commercial simulator, ANSYS HFSS. This section discusses full-wave simulation results obtained under the assumption of no material loss, and it will explain the impact of $w_{\mathrm{n}}$ and $s_{\mathrm{n}}$ on the differential-tocommon mode conversion.

\subsection{Our Bend Structures Based on Proposed Methodology}

The structural and electrical parameters are summarized in Table 1 . The dielectric constant of the glass epoxy $\varepsilon_{\mathrm{r}}$ is 4.4 , and its thickness, $h$ is $300 \mu \mathrm{m}$. The thickness of the metal used as a perfect conductor $t$ is $35 \mu \mathrm{m}$. The differential-mode characteristic impedance $Z_{\mathrm{d}}$ was set to $100 \Omega$ by using the ANSYS 2D Extractor for the cross-section stripline structure shown in Fig. 5.

Figure 6 shows the relationship between the line width and line separation in terms of the differential-mode characteristic impedance. The thick solid black lines indicate the relationship on the basis of the differential-mode characteristic impedance of $100 \Omega$. The black dot A indicates the case ( $w=0.2 \mathrm{~mm}, s=0.45 \mathrm{~mm}$ ) of the conventional bend and the

Table 1 Structural and electrical parameters of stripline.

\begin{tabular}{ccc}
\hline Item & Value & Unit \\
\hline$\varepsilon_{\mathrm{r}}$ & 4.4 & - \\
$h$ & 300 & $\mu \mathrm{m}$ \\
$t$ & 35 & $\mu \mathrm{m}$ \\
\hline
\end{tabular}

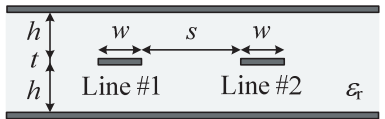

Fig. 5 Cross-sectional view of symmetric stripline.

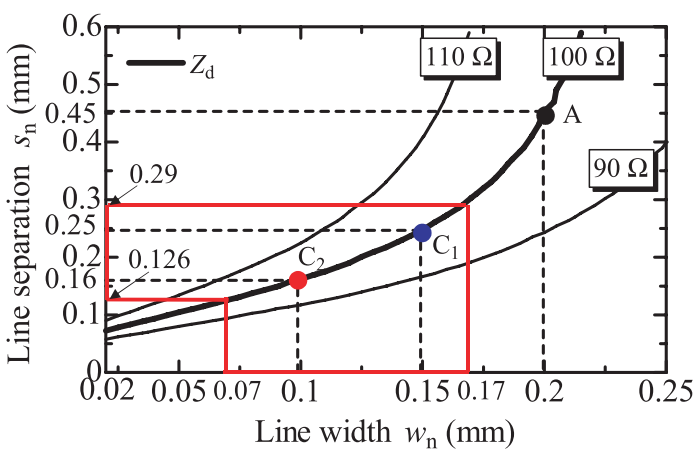

Fig. 6 Relationship between line width and line separation for differential-mode characteristic impedances of $100 \Omega$. 
Table 2 Structural parameters of bends for evaluating the impact of $w_{\mathrm{n}}$ and $s_{\mathrm{n}}$ on the differential-to-common mode conversion (unit in $\mathrm{mm}$ ), where bend $\mathrm{A}$ and bend $\mathrm{B}$ respectively correspond to the conventional bend shown in Fig. 1(a) and the tightly coupled symmetrically tapered bend shown in Fig. 1(b), and bends $\mathrm{C}_{1}$ and $\mathrm{C}_{2}$ correspond to the tightly coupled asymmetrically tapered bend shown in Fig. 1(d).

\begin{tabular}{ccccc}
\hline Item & $\mathrm{A}$ & $\mathrm{B}$ & $\mathrm{C}_{1}$ & $\mathrm{C}_{2}$ \\
\hline$w$ & 0.2 & 0.2 & 0.2 & 0.2 \\
$s$ & 0.45 & 0.45 & 0.45 & 0.45 \\
$w_{\mathrm{n}}$ & - & 0.15 & 0.15 & 0.1 \\
$s_{\mathrm{n}}$ & - & 0.25 & 0.25 & 0.16 \\
$l_{\mathrm{t}}$ & - & 0.15 & 0.15 & 0.83 \\
$l_{\mathrm{s}}$ & - & 0.5 & 0.5 & 0.5 \\
$l_{\mathrm{dg}}$ & 0.54 & 0.33 & 0 & 0 \\
\hline
\end{tabular}

common-mode characteristic impedances of $27.6 \Omega$. In accordance with the values of $w$ and $s$ of the conventional bend brought into (3), we can obtain the upper range of values $\left(w_{\mathrm{n}}\right.$ $+s_{\mathrm{n}}<0.46 \mathrm{~mm}$ ) for $w_{\mathrm{n}}$ and $s_{\mathrm{n}}$ of the tightly coupled bend. In accordance with the rules of this test board's manufacturer, the thinnest possible line width and line separation are $0.07 \mathrm{~mm}$ and $0.1 \mathrm{~mm}$, respectively. Therefore, we can obtain a range of values for $w_{\mathrm{n}}$ and $s_{\mathrm{n}}$, as shown in Fig. 6, from the area surrounded by the red line. The blue and red dots indicate the cases of the tightly coupled bends with different sets of $w_{\mathrm{n}}$ and $s_{\mathrm{n}}$, respectively. To investigate the impact of $w_{\mathrm{n}}$ and $s_{\mathrm{n}}$ on the differential-to-common mode conversion, one of them is set close to the upper limit of the value range and the other is at a lower value within the range. The blue and red dots correspond to the tightly coupled part of the tightly coupled asymmetrically tapered bend $\mathrm{C}_{1}$ (same as the tightly coupled symmetrically tapered bend $\mathrm{B}$ ) and the tightly coupled asymmetrically tapered bend $\mathrm{C}_{2}$, as shown in Table 2. Next, using the determined values of $w, s, w_{\mathrm{n}}$, and $s_{\mathrm{n}}$ into (4), we can calculate the $l_{\mathrm{t}}$ of bend $\mathrm{C}_{1}$ and bend $\mathrm{C}_{2}$, respectively. Finally, $l_{\mathrm{s}}$ is obtained in accordance with the design rules mentioned in Sect. 2.

Although the tightly coupled parts of bends $\mathrm{C}_{1}$ and $\mathrm{C}_{2}$ were designed so that the differential-mode characteristic impedance is equal to $100 \Omega$, the common-mode characteristic impedance was not controlled. The common-mode characteristic impedances in the tightly coupled part of bends $\mathrm{C}_{1}$ and $\mathrm{C}_{2}$ are 33.7 and $42.0 \Omega$, respectively.

\subsection{Evaluation of Our Bend Structures from Differential- to-Common Mode Conversion}

We evaluated the impact of $w_{\mathrm{n}}$ and $s_{\mathrm{n}}$ on the differential-tocommon mode conversion of the bend structure formed on the basis of our design methodology by full-wave simulation for four types of 45-degree-angle bend structures of which the structural parameters are listed in Table 2.

In [15], the amount of mode conversion due to the effective path difference $l_{\mathrm{d}}$ is dominant in $\left|S_{\mathrm{cd} 21}\right|$. Therefore, the mode conversion from the differential mode of the input port (Differential port 1) to the common mode of the output port (Differential port 2), $\left|S_{\mathrm{cd} 21}\right|$ is approximately [15]

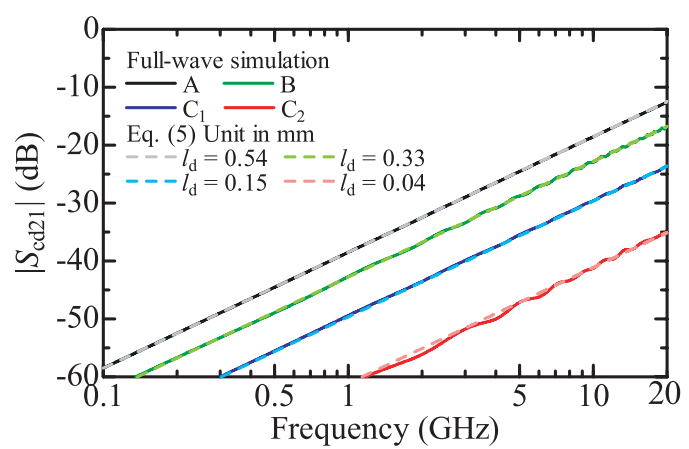

Fig. 7 Forward differential-to-common mode conversion.

Table 3 Comparison of geometrical path difference and effective path difference (unit in $\mathrm{mm}$ ).

\begin{tabular}{ccccc}
\hline Item & $\mathrm{A}$ & $\mathrm{B}$ & $\mathrm{C}_{1}$ & $\mathrm{C}_{2}$ \\
\hline$l_{\mathrm{dg}}$ & 0.54 & 0.33 & 0 & 0 \\
$l_{\mathrm{d}}$ & 0.54 & 0.33 & 0.15 & 0.04 \\
\hline
\end{tabular}

$$
\left|S_{\mathrm{cd} 21}\right| \simeq \frac{\pi \sqrt{\varepsilon_{\mathrm{reff}}}}{c} f\left|l_{\mathrm{d}}\right| .
$$

In the full-wave simulation, two differential ports were set as wave ports with the port impedances of the differential mode and common mode set to $100 \Omega$ and $25 \Omega$, respectively. The length of the coupled straight lines from Differential port 1 to the bend region is $35 \mathrm{~mm}$ and that from Differential port 2 to the bend region is $25 \mathrm{~mm}$. The dimensions of the dielectric was $60 \mathrm{~mm} \times 40 \mathrm{~mm}$.

Figure 7 shows the forward differential-to-common mode conversion coefficient $\left|S_{\mathrm{cd} 21}\right|$, and we found that the oblique tapers provide a smaller $\left|S_{\mathrm{cd} 21}\right|$ than that of symmetrically tapered bend $\mathrm{B}$, which means our bend structures can compensate for the remaining $l_{\mathrm{d}}$ in the tightly coupled bend. The broken lines indicate the respective theoretical values obtained from (5). The values of the geometrical path difference $l_{\mathrm{dg}}$ and the effective path difference $l_{\mathrm{d}}$ obtained from (5) are summarized in Table 3 . The magnitude of $\left|S_{\mathrm{cd} 21}\right|$ in bend $\mathrm{C}_{2}$ was the smallest, though all the geometrical path difference $l_{\mathrm{dg}}$ of bends $\mathrm{C}_{1}$ and $\mathrm{C}_{2}$ were set to 0 . This is because the geometrical path difference $l_{\mathrm{dg}}$ becomes less different than the effective path difference $l_{\mathrm{d}}$ with decreasing $w_{\mathrm{n}}$ and $s_{\mathrm{n}}$, and the effective path difference $l_{\mathrm{d}}$ of the smallest bend $\mathrm{C}_{2}$ is almost equal to 0 , as shown in Table 3 .

As a result, the 0.1 to $20 \mathrm{GHz}$ frequency range shows that an over $10-\mathrm{dB}$ and $20-\mathrm{dB}$ suppression of the forward differential-to-common mode conversion is achieved for $\mathrm{C}_{1}$ and $\mathrm{C}_{2}$, respectively, compared with the conventional bend.

\section{Fabrication of Bend Structures Based on Proposed Methodology and Evaluation by Measurement}

In this section, the bends with the same structural parameters (bends $\mathrm{A}, \mathrm{C}_{1}$, and $\mathrm{C}_{2}$ ) from the previous discussion were fabricated, and the differential-to-common mode conversion and differential-mode characteristics were evaluated through full-wave simulation and measurement. 


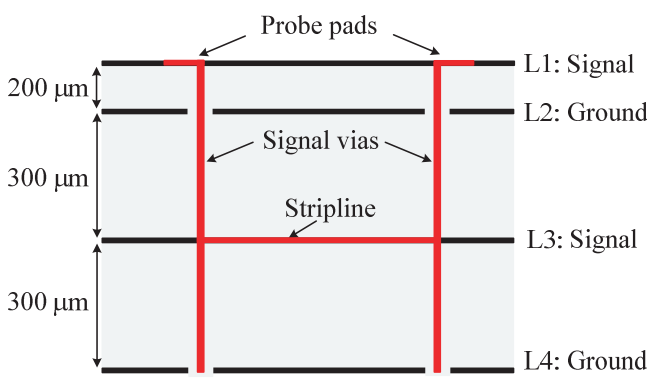

Fig. 8 Layer structure of test board.

Table 4 Common structural and electrical parameters.

\begin{tabular}{ccc}
\hline Item & Value & Unit \\
\hline$\varepsilon_{\mathrm{r}}$ & 4.4 & - \\
$\tan \delta$ & 0.02 & - \\
$\sigma$ & $5.8 \times 10^{7}$ & $\mathrm{~S} / \mathrm{m}$ \\
$h$ & 300 & $\mu \mathrm{m}$ \\
$t$ & 35 & $\mu \mathrm{m}$ \\
$Z_{\mathrm{d}}$ & 100 & $\Omega$ \\
\hline
\end{tabular}

To obtain mixed-mode S parameters, we conducted a 4-port measurement using a vector network analyzer (KEYSIGHT E5071C). We also used a pair of 200- $\mu \mathrm{m}-$ pitched GSGSG microprobes (Cascade Microtech ACP-40D-GSGSG). The measured data were plotted below $20 \mathrm{GHz}$ due to the measurement limit of the vector network analyzer.

Figure 8 shows the test board used in the actual measurements, and our bend structures to be measured are in the third layer. The lengths of the coupled straight lines from Differential ports 1 and 2 to the bend region are 35 and $25 \mathrm{~mm}$, respectively. Copper was used as the conductor, and a glass epoxy material was used between the layers. Each terminal of the stripline structure (differential transmission lines) was connected to the probe pads by using through-hole signal vias.

Table 4 summarizes the common structural and electrical parameters. To compare the results with those of the measurement, dielectric loss $\tan \delta$ and copper conductivity $\sigma$ were taken into account in the full-wave simulation. The probe pads and the through-hole signal vias (using the same size as the design drawing) were added on the two-terminal of the differential transmission lines. Each port was set as a lumped port with the port impedances of the differential mode set to $100 \Omega$ and that of the common mode to $25 \Omega$. The dimensions of the dielectric was $75 \mathrm{~mm} \times 40 \mathrm{~mm}$.

Figure 9(a) shows the forward differential-to-common mode conversion $\left|S_{\mathrm{cd} 21}\right|$ as a function of frequency concerning bends $\mathrm{A}, \mathrm{C}_{1}$, and $\mathrm{C}_{2}$. The solid and broken lines indicate the measurement and full-wave simulation results, respectively. The results obtained from the full-wave simulation are almost in agreement with those of the measurement. These measurement results confirm that the difference between the geometric path difference and the effective path difference is reduced by decreasing the line width $w_{\mathrm{n}}$ and line separation $s_{\mathrm{n}}$ of the tightly coupled bend. The forward differential-to-common mode conversion $\left|S_{\mathrm{cd} 21}\right|$ of bend $\mathrm{C}_{2}$

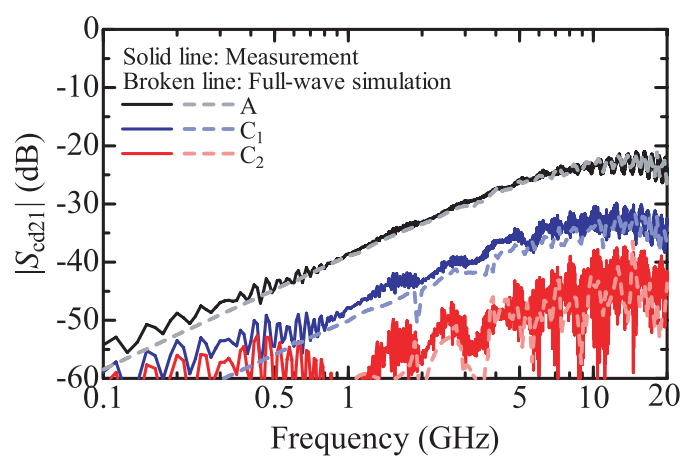

(a) Forward differential-to-common mode conversion

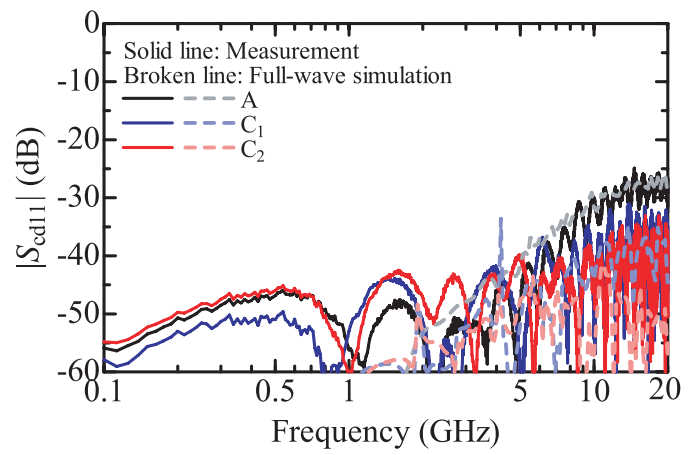

(b) Backward differential-to-common mode conversion

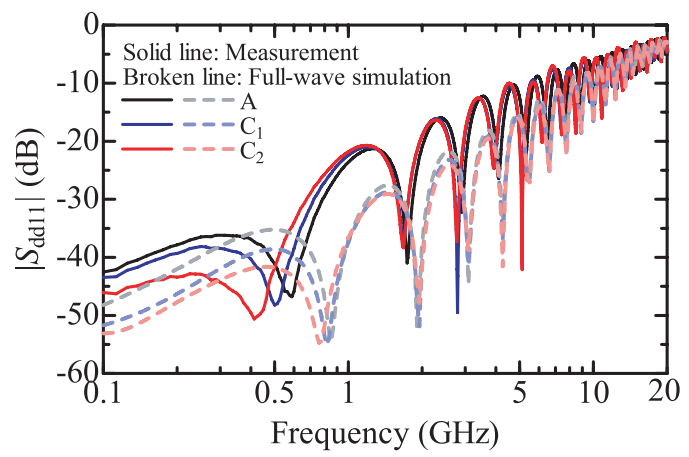

(c) Differential-mode reflection coefficient

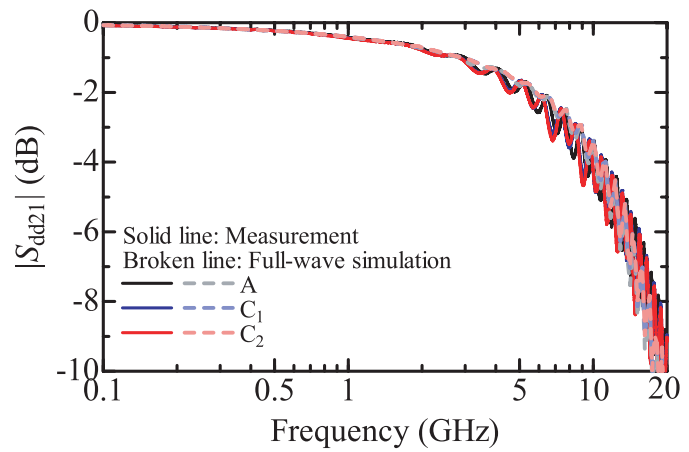

(d) Differential-mode transmission coefficient

Fig. 9 Comparisons of mode-conversion and differential-mode characteristics obtained by full-wave simulation and measurement.

was decreased by approximately $20 \mathrm{~dB}$ compared with that of bend A. Next, Fig. 9(b) shows the backward differential-tocommon mode conversion $\left|S_{\mathrm{cd} 11}\right|$ as a function of frequency. At over $5 \mathrm{GHz}$, the backward differential-to-common mode 
conversion of bends $\mathrm{C}_{1}$ and $\mathrm{C}_{2}$ is smaller compared with that of bend $\mathrm{A}$. This means that our bend structures produce a smaller amount of differential-to-common mode conversion than bend A. As shown in Fig. 9(c), our design methodology helps maintain its differential-mode reflection $\left|S_{\mathrm{dd} 11}\right|$ compared with that of the conventional bend. The increase of $\left|S_{\mathrm{dd} 11}\right|$ at higher frequencies is a result of the effect of the probe pads. For the differential-mode transmission coefficient, as shown in Fig. 9(d), the results obtained from the full-wave simulation are almost in agreement with those of the measurement, and the bends $C_{1}$ and $C_{2}$ are comparable with bend A.

\section{Conclusion}

In this paper, we proposed the practical and straightforward design methodology of our tightly coupled asymmetrically tapered bend to limit the bend to within the area of the original classic bend for high-density differential wiring. Fullwave simulations indicated that the amount of the mode conversion is close to the expected value by making $w_{\mathrm{n}}$ and $s_{\mathrm{n}}$ as small as possible. In addition, full-wave simulation and measurement results demonstrated that the bend structure formed on the basis of our design methodology sufficiently suppresses the differential-to-common mode conversion and provides similar differential-mode transmission characteristics compared with the conventional bend structure.

\section{Acknowledgments}

This study was partially supported by Grant-in-Aid for Scientific Research (B) 16H04318.

\section{References}

[1] G.H. Shiue, W.D. Guo, C.M. Lin, and R.B. Wu, "Noise reduction using compensation capacitance for bend discontinuities of differential transmission lines," IEEE Trans. Adv. Packag., vol.29, no.3, pp.560-569, Aug. 2006.

[2] T.L. Wu, F. Buesink, and F. Canavero, "Overview of signal integrity and EMC design technologies on PCB: fundamentals and latest progress," IEEE Trans. Electromagn. Compat., vol.55, no.4, pp.624-638, Aug. 2013.

[3] C.H. Chang, R.Y. Fang, and C.L. Wang, "Bended differential transmission line using compensation inductance for common-mode noise suppression,” IEEE Trans. Compon., Packag., Manuf. Technol., vol.2, no.9, pp.1518-1525, Sept. 2012

[4] L.S. Wu, J.F. Mao, and W.Y. Yin, "Slow-wave structure to suppress differential-to-common mode conversion for bend discontinuity of differential signaling," Proc. IEEE Elect. Design Adv. Packag. Syst. Symp., pp.219-222, Taipei, China, Dec. 2012.

[5] D.B. Lin, C.P. Huang, and H.N. Ke, "Using stepped-impedance lines for common-mode noise reduction on bended coupled transmission lines," IEEE Trans. Compon., Packag., Manuf. Technol., vol.6, no.5, pp.757-766, May 2016.

[6] T. Matsushima and O. Wada, "Imbalance control by open stub for reduction of common-mode conversion at differential transmission line bend," Proc. EMC'14/Tokyo, pp.125-128, May 2014.

[7] T. Matsushima and O. Wada, "Reduction of common-mode excitation on a differential transmission line bend by imbalance control," IEICE Communications Express, vol.3, no.10, pp.295-299, 2014.
[8] B.R. Huang, C.H. Chang, R.Y. Fang, and C.L. Wang, "Commonmode noise reduction using asymmetric coupled line with SMD capacitor," IEEE Trans. Compon., Packag., Manuf. Technol., vol.4, no.6, pp.1082-1089, June 2014.

[9] S. Lee, J. Lim, S. Oh, Y. Kim, D. Oh, and J. Lee, "Differential-tocommon-mode conversion suppression using mushroom structure on bent differential transmission lines," IEEE Trans. Compon., Packag., Manuf. Technol., vol.9, no.4, pp.702-711, April 2019.

[10] C. Gazda, D.V. Ginste, H. Rogier, R.-B. Wu, and D. De Zutter, "A wideband common-mode suppression filter for bend discontinuities in differential signaling using tightly coupled microstrips," IEEE Trans. Adv. Packag., vol.33, no.4, pp.969-978, Nov. 2010.

[11] C. Gazda, D.V. Ginste, H. Rogier, D. De Zutter, and R.-B. Wu, "Time domain analysis of a wideband common-mode suppression filter for bent interconnects," Proc. 2011 IEEE 15th Workshop on Signal Propagation on Interconnects (SPI), pp.7-10, May 2011.

[12] C. Gazda, I. Couckuyt, H. Rogier, D.V. Ginste, and T. Dhaene, "Constrained multiobjective optimization of a common-mode suppression filter," IEEE Trans. Electromagn. Compat., vol.54, no.3, pp.704-707, June 2012.

[13] C. Gazda, H. Rogier, D.V. Ginste, I. Couckuyt, and T. Dhaene, "Time domain analysis of a common-mode suppression filter subjected to a multi-objective optimization,” Proc. EMC Europe 2012, pp.17-21, Sept. 2012

[14] S. Kan, Y. Toyota, K. Iokibe, and T. Watanabe, "Suppression of mode conversion by decreasing path difference by using an asymmetrically tapered bend in differential transmission lines," Proc. EMC'14/Tokyo, pp.129-132, May 2014.

[15] Y. Toyota, S. Kan, and K. Iokibe, "Suppression of mode conversion by using tightly coupled asymmetrically tapered bend in differential lines," IEICE Trans. Commun., vol.E98-B, no.7, pp.1188-1195, July 2015.

[16] C. Wang, K. Iokibe, and Y. Toyota, "Tightly coupled asymmetrically tapered bend in differential transmission lines for high-density mounting," IEICE Technical Report, EMCJ2015-39, July 2015 (in Japanese).

[17] C.Y. Wang, K. Iokibe, and Y. Toyota, "Design methodology of tightly coupled asymmetrically tapered bend for high-density mounting in differential transmission lines," 2016 Asia-Pacific International Symposium on Electromagnetic Compatibility \& Signal Integrity and Technical Exhibition (APEMC 2016), Shenzhen, China, pp.463465, May 2016.

[18] S.H. Hall, G.W. Hall, and J.A. McCall, High-Speed Digital System Design: A Handbook of Interconnect Theory and Design Practices, Wiley, New York, NY, USA, 2000.

[19] F.D. Mbairi, W.P. Siebert, and H. Hesselbom, "High-frequency transmission lines crosstalk reduction using spacing rules," IEEE Trans. Compon. Packag. Technol., vol.31, no.3, pp.601-610, 2008.

\section{Appendix: Derivation Process of (3) and (4)}

Figure A. 1 shows the asymmetrically tapered enlarged view. The necessary structural parameters are as follows: the center-line lengths $l_{\mathrm{t} 1}$ and $l_{\mathrm{t} 2}$ of the tapers of Line \#1 and Line \#2, respectively, result in the compensation amount $\Delta l_{\mathrm{t}}$ as follows

$$
\Delta l_{\mathrm{t}}=l_{\mathrm{t} 2}-l_{\mathrm{t} 1} \quad\left(l_{\mathrm{t} 2}>l_{\mathrm{t} 1}\right) .
$$

Figure A. 1 shows $l_{\mathrm{a}}$ as follows

$$
l_{\mathrm{a}}=(w+s)-\left(w_{\mathrm{n}}+s_{\mathrm{n}}\right),
$$

and, $l_{\mathrm{b}}$ is a required constraint condition for limiting the asymmetrically tapered bend into the area of the conventional 


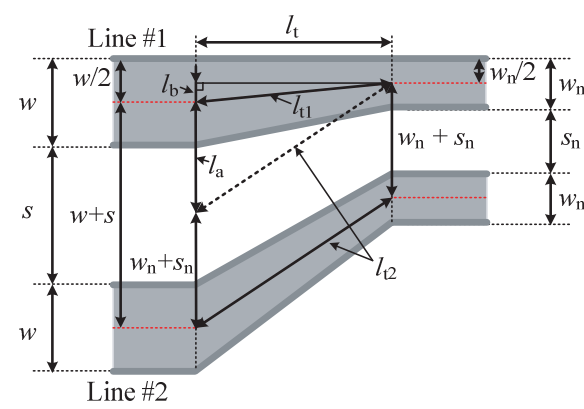

Fig. A. 1 Asymmetrically tapered enlarged view.

bend as follows:

$$
l_{\mathrm{b}}=\frac{w-w_{\mathrm{n}}}{2} \text {. }
$$

As seen in Fig. A 1 , three sides of $l_{\mathrm{t} 1}, l_{\mathrm{t} 2}$, and $l_{\mathrm{a}}$ form a triangle. Thus, the length of one side must be greater than the difference between the lengths of the other two sides due to the conditions for the establishment of triangles, so the range that $\Delta l_{\mathrm{t}}$ can take is given by

$$
\Delta l_{\mathrm{t}}<l_{\mathrm{a}}
$$

Here, for an asymmetric taper, $\Delta l_{\mathrm{t}}>0$.

Substituting (A. 1) and (A. 2) into (A.4) produces the following equation

$$
\left(w_{\mathrm{n}}+s_{\mathrm{n}}\right) \tan \left(\frac{\phi}{2}\right)-\frac{l_{\mathrm{dg}}}{2}<(w+s)-\left(w_{\mathrm{n}}+s_{\mathrm{n}}\right)
$$

Here, the geometric path difference $l_{\mathrm{dg}}=0$ is a condition, and (A. 5) can be rewritten as (3).

In Fig. A. 1, there are two right-angled triangles, one consisting of $l_{\mathrm{t} 1}, l_{\mathrm{t}}$, and $l_{\mathrm{b}}$, and the other consisting of $l_{\mathrm{t} 2}$, $l_{\mathrm{t}}$, and $\left(l_{\mathrm{a}}+l_{\mathrm{b}}\right)$. Therefore, we can produce the following formulas

$$
\begin{aligned}
& l_{\mathrm{t} 1}^{2}=l_{\mathrm{t}}^{2}+l_{\mathrm{b}}^{2} \\
& l_{\mathrm{t} 2}^{2}=l_{\mathrm{t}}^{2}+\left(l_{\mathrm{a}}+l_{\mathrm{b}}\right)^{2}
\end{aligned}
$$

Substituting (A. 2), (A. 3), (A. 6), and (A. 7) into (A. 1), we obtain (4).

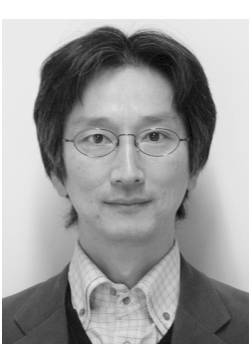

Kengo Iokibe received B.S., M.S., and Ph.D. degrees in Electrical and Electronic Engineering from Okayama University in 1997, 1999, and 2005, respectively. He was a Research Associate in the Department of Communication Network Engineering, Okayama University, from 2000 to 2002. He is currently an Assistant Professor in the Department of Information and Communication Systems, Graduate School of Natural Science and Technology, Okayama University. His recent research interests include information security against side-channel attacks on cryptographic circuits, designing power distribution networks to achieve PI, SI and EMC, and EMC modeling of power converters. Dr. Iokibe is a senior member of the Institute of Electronics, Information and Communication Engineers and the Japan Institute of Electronics Packaging.

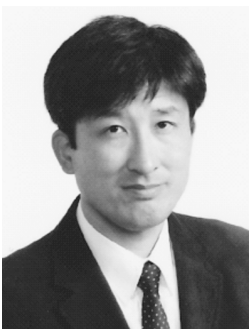

Yoshitaka Toyota received B.S. and M.S degrees in Electrical and Electronic Engineering from Okayama University in 1991 and 1993, respectively, and a Ph.D. degree in Electronic Engineering from Kyoto University, Kyoto, Japan, in 1996. From 1996 to 1998 , he was with Yokogawa Electric Company, Ltd. and in 2005 he worked at Georgia Tech as an Overseas Research Scholar of the Ministry of Education, Culture, Sports, Science and Technology (MEXT) of Japan. He is currently a Professor of the Graduate School of Natural Science and Technology, Okayama University. His recent research interests include EMC design for high-speed digital systems. Prof. Toyota is a senior member of the Institute of Electronics, Information and Communication Engineers, the Japan Institute of Electronics Packaging, and the Japan Society of Applied Physics.

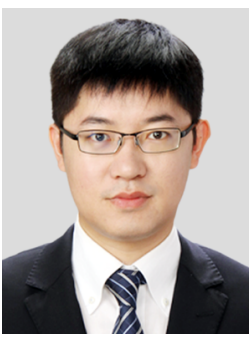

Chenyu Wang received the M.S and Ph.D. degrees in electrical and communication engineering from Okayama University, Okayama, Japan, in 2017 and 2020, respectively. His research interests include high-quality and low noise transmission in the high-speed transmission system of PCB. He is currently working for KYOCERA Corporation. Dr. Wang is a member of the Institute of Electronics, Information and Communication Engineers. 